Review

\title{
Factors to Consider When Assessing the Diagnostic Accuracy of Synovial Leukocyte Count in Periprosthetic Joint Infection
}

\author{
Karsten D Ottink ${ }^{\circledR}$, Carol Strahm², Anneke Muller-Kobold 3 , Parham Sendi ${ }^{4,5,6}$, Marjan \\ Wouthuyzen-Bakker7 \\ 1. Department of Orthopaedics, University of Groningen, University Medical Centre Groningen, the Netherlands \\ 2. Division of Infectious Diseases and Hospital Epidemiology, Cantonal Hospital St. Gallen, Switzerland \\ 3. Department of Laboratory Medicine, University of Groningen, University Medical Centre Groningen, The Netherlands \\ 4. Department of Infectious Diseases and Hospital Epidemiology, University Hospital Basel, University Basel, Basel, Switzerland \\ 5. Department of Orthopaedics and Traumatology, University Hospital Basel, Basel, Switzerland. \\ 6. Institute for Infectious Diseases, University of Bern, Bern, Switzerland. \\ 7. Department of Medical Microbiology and Infection Prevention, University of Groningen, University Medical Centre Groningen, The Netherlands \\ $\triangle$ Corresponding author: Karsten Ottink, Department of Orthopaedic Surgery, University Medical Center Groningen, Groningen the Netherlands. Email: \\ karstenottink@gmail.com
}

(C) The author(s). This is an open access article distributed under the terms of the Creative Commons Attribution License (https://creativecommons.org/licenses/by/4.0/). See http://ivyspring.com/terms for full terms and conditions.

Received: 2019.03.13; Accepted: 2019.06.05; Published: 2019.08.02

\begin{abstract}
Synovial white blood cell (WBC) count and the percentage of polymorphonuclear leucocytes (PMN\%) is one of the diagnostic criteria to diagnose a periprosthetic joint infection (PJI). Although the test is widely available, the diagnostic accuracy of proposed cut-off levels are influenced by several factors, such as: the affected joint, co-morbid conditions, the causative microorganism and the gathering and processing of samples in the laboratory. In this narrative review we provide an overview on how and to what extent these factors can affect the synovial WBC count and PMN\% in synovial fluid.
\end{abstract}

Key words: periprosthetic joint infection, synovial fluid, diagnosis, white blood cell count, polymorphonuclear percentage

\section{Introduction}

The diagnosis of a periprosthetic joint infection (PJI) is challenging especially for chronic PJIs. Because there is no single and absolute test to confirm or exclude infection [1-7], patients' history, physical examination, joint specific $\mathrm{x}$-ray or other types of imaging, histology, culture and inflammatory markers should all be taken into consideration for diagnosis. It is critical to distinguish between septic and aseptic failure preoperatively, as arthroplasty exchange of a missed PJI leads to subsequent failure $[8,9]$. Criteria for the diagnosis of PJI have been proposed by the Musculoskeletal Infection Society (MSIS) and the International Consensus Meeting
(ICM) [10-12]. One of them includes the synovial white blood cell (WBC $>3000$ cells $/ \mu \mathrm{L}$ ) count and its proportion of polymorphonuclear cells $(\mathrm{PMN} \%>70)$. The test can be performed relatively easily in the preoperative diagnostic work-up. Its sensitivity, specificity and diagnostic accuracy depends on the threshold that is applied. Factors such as joint site, co-morbid conditions, the causative microorganism and the duration of infection, in addition to pre-analytical laboratory variables, may influence the optimal cut-off result [13]. This narrative review provides an overview of these factors and its possible influence on the threshold and accuracy of the WBC 
count and PMN\% in synovial fluid for PJIs.

\section{Search strategy and selection criteria}

We identified references for this narrative review through searches of PubMed and Embase electronic databases by using the following combinations of terms (alternative terms grouped in parentheses): ("synovial" OR "joint fluid" OR "synovial fluid") AND ("white blood cell" OR "WBC" OR "leucocyte" OR "polymorphonuclear" OR "PMN") AND ("count" OR “differentiation" OR "analysis")). We included publications from January 1966 to December 2018. The title and abstract were screened to identify the references applicable to the different research questions of this review, after which the full text articles were evaluated.

\section{Historical perspective: from native to prosthetic joints}

Synovial fluid analysis has traditionally been considered in native joints to differentiate between non-inflammatory (typically with a WBC count of < 2,000 cells $/ \mu \mathrm{L}$ and $\mathrm{PMN} \%$ of $<25$ ) and inflammatory arthritis[14,15]. In a systematic review on native septic arthritis, synovial WBC count of $>25,000$ cells $/ \mu \mathrm{L}$, $>50,000$ cells $/ \mu \mathrm{L}$ and $>100,000$ cells $/ \mu \mathrm{L}$ revealed a sensitivity of $77 \%, 62 \%$ and $29 \%$ and a specificity of $73 \%, 92 \%$ and $99 \%$, respectively[16].

The diagnostic use of joint aspiration before revision arthroplasty was first described by Duff et al. [17] in 1996. The authors retrospectively reviewed the preoperative aspiration results of 43 knees between 1985 and 1995. They investigated microbiological growth but did not report results of WBC count analyses. Spangehl et al. [18] prospectively analysed the WBC count in synovial fluid in 183 cases during revision of total hip arthroplasties (THAs) from 1994 to 1996. The authors considered it a positive finding if the WBC count was $>50,000$ cells/ $\mu \mathrm{L}$ or the PMN\% was $>80 \%$ (i.e.; cut-offs used for native septic arthritis). They reported a good specificity of $99 \%$ $(96 \%-100 \%)$ but a poor sensitivity of 36\% (19\%-56\%). Kersey et al. [19] reported a mean WBC count of 782 cells $/ \mu \mathrm{L}$ (11 to $7,200 / \mu \mathrm{L})$ and a PMN\% of 13 in 77 total knee arthroplasties (TKAs) undergoing revision for 'aseptic failure' from 1992 to 1997. The authors calculated that a WBC count of $<2,000 / \mu \mathrm{L}$ and a $\mathrm{PMN} \%$ of $<50$ had a $98.3 \%$ negative predictive value for excluding infection.

In 2003, Mason et al. [20] demonstrated an important finding for the diagnosis of PJI. The specificity for diagnosing PJI with a WBC count cut-off value of 50,000 cells $/ \mu \mathrm{L}$ is similar to the specificity of using a value of 2,500 cells $/ \mu \mathrm{L}$. They retrospectively reviewed preoperative aspiration data of 86 knees from 1986 to 1997 and calculated a specificity of $95 \%$ and sensitivity of $98 \%$ for diagnosing PJI when using a WBC count cut-off value of 2,500 cells $/ \mu \mathrm{L}$ combined with a PMN\% of $>60$. After Trampuz et al. [21] (1,700 cells/ $\mu \mathrm{L}$ for knee PJI) and Schinsky et al. [22] (3,000 to 4,200 cells/ $\mu$ L for hip PJI) reported similar findings in 2004 and 2008, respectively, it was apparent that considerably lower WBC count cut-off values should be used for the diagnosis of PJI than for the diagnosis of native septic arthritis.

\section{The confusion about the units}

The observation that lower cut-off levels for the synovial WBC count were necessary in prosthetic joints than in native joints (i.e., more than $1 \mathrm{log}$ ), together with the use of different units, can cause some confusion in the nomenclature. The frequently quoted unit is cells $/ \mu \mathrm{L}$ [23], equivalent to cells $/ \mathrm{mm}^{3}$, $10^{6}$ cells/ $\mathrm{L}$ or cells $/ 10^{-3} \mathrm{~cm}^{3}$. The units may vary between institutions, depending on laboratory practice. Thus, attention to detail is required when converting the results from one unit to another to prevent errors [24].

\section{Factors potentially influencing synovial WBC count thresholds}

Synovial WBC count cut-off values ranging from 1,000 to 5,000 cells $/ \mu$ for the diagnosis of chronic hip and knee PJI have been published. Subsequently, there is an ongoing discussion about the optimal cut-off value. For simplicity, surgeons and physicians like to use a single optimal cut-off value. However, several variables may influence the final result, as discussed in the following paragraphs.

\section{Preanalytical steps}

- USE OF LOCAL ANAESTHETICS: The influence of local anaesthetics on synovial fluid WBC has not been studied. However, local anaesthetics and related compounds may inhibit the growth of bacteria, presumably because of the acidity of most anaesthetic compounds. Therefore, local anaesthetics prior to joint puncture is discouraged [25].

- PRIOR USE OF ANTIBIOTICS: Lower WBC counts and PMN\% in synovial fluid have been found in patients treated with antimicrobial therapy than in patients without antibiotic therapy [26]. Therefore, antibiotics should be withheld in suspected chronic PJIs until a proper diagnostic work-up has been performed.

- VOLUME: The optimal synovial fluid volume 
for analyses of WBC count is typically $1 \mathrm{~mL}$ or more. However, depending on the analytical procedure, smaller volumes are possible (e.g., $250 \mu \mathrm{L}$ ). It is important to interact closely with laboratory experts to clarify these details, since there are inter-institutional differences in standard operating procedures between laboratories.

- DRY TAP: In case of a punctio sicca ("dry tap"), injection and re-aspiration of sodium chloride may be helpful to obtain bacterial culture. However, WBC analysis is not reliable anymore and should not be performed. Of note, dry tap does not exclude an infection, and the frequency of dry taps may vary between joints. In a study by Zahar et al. [27], a complete synovial fluid analysis was available in only $64 \%$ of all studied patients with a hip or knee arthroplasty joint due to a limited amount of synovial fluid, a clotted specimen or a bloody aspirate.

- CLOTTING: To prevent clotting, synovial fluid should be collected in ethylenediaminetetraacetate (EDTA) tubes. The tube should be turned upside down several times to mix the EDTA with the synovial fluid. Mucous samples are due to its increased viscosity - difficult to pipet. The viscosity may also form a problem for automated cell analysers in aspirating a sufficient amount of fluid. Pre-treatment of the sample with hyaluronidase reduces the viscosity of synovial fluid and improves the accuracy of cytometric analysis, irrespective of whether optical or automated techniques are used [28]. Of note, an EDTA tube is not preferred for crystal examination.

- BLOODY ASPIRATE: In the case of an aspirate with a high number of red blood cells (RBCs), it is possible to calculate an approximation of corrected values for the synovial WBC count by using a mathematical model and the serum WBC count [29]. The adjusted synovial WBC count can be calculated with the following formula: synovial $\mathrm{WBC}$ adjusted $=$ synovial $\mathrm{WBC}$ observed [(WBC blood / RBC blood $) \times R B C$ synovial fluid] [30]. Discussion of the results with a laboratory expert is advised in these cases.

- TRANSPORT: Ex vivo cell lysis may occur during prolonged transport. Rapid transport of the sample to the laboratory with swift analysis of the material may prevent cell lysis. The optimal time interval has not been investigated for patients with PJI. We recommend analysis within 1 hour, preferentially within 30 minutes, of aspiration.
- LABORATORY CELL COUNTING: Studies have demonstrated that standardized automated counting of WBCs in synovial fluid is more accurate and efficient than manual counting [31-33]. Manual counting of WBCs in synovial fluid results in an inter-observer variance of $>20 \%$ $[28,31,34]$. In case of frank pus or metal on metal arthroplasty, the sample consists of high numbers of necrotic and apoptotic leucocytes. These cellular debris may result in false results in automated systems. In such a scenario, samples should be manually counted by a laboratory technician.

\section{Joint site}

The location of the affected joint has an effect on the optimal threshold of the WBC count and the $\mathrm{PMN} \%$. A possible hypothesis for this is because of the differences in joint space/volume, synovial lining and vascularization. All of these factors theoretically may be influence the concentration of WBC and PMN\% in a joint.

- HIP: Several studies investigated WBC count and PMN\% in PJI diagnosis of the hip [27,35-45]. These studies indicate an optimal cut-off for the WBC count of 3,000 cells/ $\mu \mathrm{L}$ and a PMN\% of 65 . This cut-off value was supported by a recent meta-analysis that demonstrated subsequent sensitivity and specificity of $90 \% \quad(95 \%$ confidence interval [CI] 0.828-0.938) and 86\% (95\% CI 0.704-0.939), respectively, for the WBC count and of $88 \%$ (95\% CI $0.818-0.927)$ and $82 \%(95 \%$ CI 0.759-0.870), respectively, for PMN\%.

- KNEE: We reviewed 15 studies that investigated the WBC count and PMN\% in PJI diagnosis of the knee $[27,35,37,38,42,46-55]$. In a meta-analysis performed by De Fine et al., a higher diagnostic accuracy was observed in TKA PJI compared to THA PJI (Table 1) when using a threshold of 3,000 cells $/ \mu \mathrm{L}$ and a PMN\% of $>65$ [55]. It should be noted that due to the lack of an adequate number of clinical studies specifically involving TKAs, the precise assessment of the optimal cut-off threshold for TKA PJI was precluded in the meta-analysis [55]. Zahar et al. [27] performed a single-centre retrospective study that included 179 patients with TKA. The authors calculated an optimal cut-off threshold for the WBC count of 1,630 cells $/ \mu \mathrm{L}$ with a sensitivity of $84 \%$ and specificity of $82 \%$. The optimal cut-off for PMN\% was set at 61, resulting in a sensitivity of $80 \%$ and a specificity of $77 \%$ [27]. Together with the previously mentioned data of Mason et al. [20] and Trampuz et al. [21], the results tended to demonstrate 
lower cut-off levels in knee joints than in hip joints.

- SHOULDER: Only a few studies have evaluated the diagnostic accuracy of the WBC count and PMN\% in shoulder arthroplasties, and all of these studies comprised small cohorts [56,57]. Recently, Strahm et al. [58] analysed 19 failed shoulder implants in which a WBC count and PMN\% was performed prior to revision surgery. Sixteen of these cases $(84 \%)$ were diagnosed with infection; the optimal synovial WBC count threshold was 12,200 cells $/ \mu L$ (sensitivity $92 \%$ and specificity 100\%) and the optimal PMN\% threshold was 54 (sensitivity 100\% and specificity 75\%). This higher threshold for infection in shoulders compared with hips and knees was also observed in previous reports [59]. These data indicate that a different threshold should be applied for shoulder arthroplasties, but larger studies are needed to confirm these findings.

- ANKLE AND ELBOW: The joint volume of the ankle and elbow is limited. This is particularly the case after previous surgery and the presence scar tissue. Consequently, synovial fluid can rarely be aspirated and no published data is available about the diagnostic accuracy of WBC count and PMN\% in the ankle [60] or elbow [61].

Table 1. Synovial fluid analysis in THA vs TKA according to the meta-analysis of De Fine et al. [55].

\begin{tabular}{lllll}
\hline WBC count 3,000 cells/ $\boldsymbol{\mu L}$ & Sensitivity & $\mathbf{9 5 \%} \mathbf{C I}$ & Specificity & $\mathbf{9 5 \%} \mathbf{C I}$ \\
\hline THA & 0.89 & $0.83-0.93$ & 0.86 & $0.72-0.93$ \\
TKA & 0.90 & $0.77-0.96$ & 0.94 & $0.63-0.99$ \\
& & & & \\
\hline PMN\% > 65 & Sensitivity & $\mathbf{9 5 \%}$ CI & Specificity & $\mathbf{9 5 \%}$ CI \\
\hline THA & 0.88 & $0.82-0.93$ & 0.82 & $0.75-0.88$ \\
TKA & 0.93 & $0.80-0.98$ & 0.90 & $0.76-0.97$ \\
\hline
\end{tabular}

\section{Co-morbid conditions}

It is conceivable that co-morbid conditions and immunosuppressive drugs have an influence on the production and function of leucocytes.

- INFLAMMATORY ARTHRITIS: Because an inflammatory arthritis causes an increase in synovial WBC [62], it is reasonable to assume a different treshold in a rheumatic than in a non-rheumatic joint with PJI. However, the study by Cipriano et al. [37] indicated that there is no clear difference in thresholds for the WBC count and PMN\% for diagnosing a PJI in patients with and without inflammatory arthritis. Indeed, the meta-analysis performed by Qu et al. [63], in which the authors compared 8 studies that excluded patients with inflammatory arthritis $(n=2102)$ with 6 studies that included patients with inflammatory arthritis $(n=598)$, demonstrates no differences in the diagnostic accuracy of synovial fluid WBC count and PMN\% between groups. Because of the high heterogeneity of the included studies, the authors were not able to determine the optimal cut-off values in the meta-analysis [63]. In a multicentre study performed by Shohat et al. [64] that included 1,220 patients undergoing revision arthroplasty, thresholds for WBC count associated with PJI in patients with and without inflammatory arthritis were 2,533 and 2,683 cells $/ \mu \mathrm{L}$, respectively, and 73 and 72 for PMN\%. Notably, in this analysis, the sensitivity was approximately $8 \%$ higher and the specificity approximately $15 \%$ lower in patients with inflammatory arthritis than in patients without inflammatory arthritis. However, the same thresholds were applied for both patient categories. The use of immunosuppressive drugs were not taken into account in these studies.

- METAL-ON-METAL (MoM) PROSTHESIS: In MoM prosthesis, metal wear from either the trunnion between the stem and the head or from the joint line of the THA, causes free metal particles. These particles may cause lymphocyte-dominated vasculitis-associated lesions (ALVAL) [40]. Both the free metal particles and the subsequent inflammatory reaction make the synovial fluid analysis more difficult to perform, and also, to interpret. The diagnosis of PJI in patients with failed MoM bearings and/or corrosion reactions in hip arthroplasties is challenging [65]. The clinical presentation can mimic a PJI, and aspiration of joint fluid may demonstrate pus [66]. Hence, analysis of synovial fluid sample is not possible in these cases, because of the presence of metal or amorphous material, fragmented cells and/or clots. A manual count may identify the presence of metal or amorphous material. Also, the synovial WBC and PMN\% are false positive for infection in these cases [66]. However, the proportion of these misleading results in patients with MoM is difficult to assess [40,44]. Several studies demonstrated a relatively high diagnostic accuracy of synovial WBC count and PMN\% for the diagnosis of PJI in patients with MoM. In a study including 102 MoM THAs, the optimal cut-off threshold for the WBC count in diagnosing PJI was 3,111 cells/ $\mu \mathrm{L}$ (sensitivity 
$94 \%$, specificity $86 \%$ ), and thus, similar to non-MoM prostheses. The optimal cut-off for PMN\% was 85 with a sensitivity of $82 \%$ and specificity of $87 \%$ [66].

- CRYSTAL-INDUCED ARTHRITIS IN ARTHROPLASTY: Crystal-induced arthritis (gout, pseudogout) in a periprosthetic joint is a rare phenomenon [67-70]. Analysis for crystal disease should always be included in the diagnostic workup of PJI, in particular in the case of predisposing factors for (pseudo) gout, such as alcohol abuse, renal failure or the use of diuretics, especially in patients with an acute presentation of arthritis [71]. Crystal-induced arthritis may present with clinical manifestations that are identical to those of a PJI [72], and high WBC counts are reported in cases of acute gout (e.g.; up to 500,000 cells $/ \mu \mathrm{L}$ ) [73]. It should be noted that the presence of crystal arthritis does not rule out a concurrent PJI. Patients with crystal-induced diseases may have a incidence of PJIs than patients without gout or pseudogout $[74,75]$.

\section{Causative microorganism and bacterial inoculum}

The microorganisms causing PJI largely depend on the type of infection (acute or chronic). Most diagnostic studies that evaluate the WBC count do not describe the cultured microorganisms. It is reasonable to assume that low-grade microorganisms, such as Cutibacterium acnes, cause a different or limited immunological response in comparison to that of more virulent microorganisms, like Staphylococcus aureus, Streptococcus species or Gram negatives [76,77]. Indeed, it has been demonstrated that the sensitivity of several (synovial) biomarkers in C. acnes PJIs are moderate to poor [77,78]. Recently, Kheir et al. [79] described the accuracy of several diagnostic tests per causative microorganism in 549 chronic PJIs and 653 aseptic revisions. The optimal cut-off for the WBC count was around 2,700-3,000 cells $/ \mu \mathrm{L}$ irrespective of the causative microorganism, but appeared to be lower for Enterococcus species (optimal cut-off of around 1,700 cells $/ \mu \mathrm{L})$. The same applied to the PMN \%, which was $\sim 65$ for most microorganisms, but $\sim 58$ for Enterococcus species. Notably, only 14 PJIs caused by enterococci were included in the study and infections caused by $C$. acnes were not reported in this cohort.

\section{Currently applied thresholds for WBC count in synovial fluid}

Different institutions have different practices regarding the use of one cut-off level for all joints or the use of joint-specific cut-off levels. In the latter case, providers frequently use WBC count cut-offs of 1,100 to 1,700 cells/ $\mu \mathrm{L}$ for knee PJI and 3,000 to 4,200 for hip PJI [27,53,55]. Renz et al. [80] suggested $>2,000$ cells $/ \mu \mathrm{L}$ and $>70 \%$ PMN for both joint sites and demonstrated a sensitivity of $85.5 \%$ (95\% CI 75.6-92.6) and a specificity of $92.3 \%$ (95\% CI 86.3-96.3). In a meta-analysis, a cut-off threshold for WBC count of $>3,000$ cells $/ \mu \mathrm{L}$ (irrespective of joint site) was calculated to have the highest sensitivity of $89 \%$ (95\% CI $0.86-0.91$ ) and specificity of $86 \%$ (95\% CI $0.80-0.90)$ [55]. A cut-off threshold for PMN\% of $>65$ was calculated to have the highest sensitivity of $89 \%$ (95\% CI $0.82-0.93$ ) and specificity of $86 \%$ (95\% CI 0.77-0.92) [81]. A recent study by Zahar et al. [27] solely analysed patients with a chronically painful hip and knee arthroplasty; data on the WBC count was available in 337 cases. The proposed cut-off in this study was in accordance with the previously proposed thresholds for infection of $>3,000$ cells $/ \mu \mathrm{L}$ and a PMN\% of 65 . During the second International Consensus Meeting on Musculoskeletal Infections in Philadelphia, the agreed threshold for WBC count was $>3,000$ cells $/ \mu \mathrm{L}$ and a $\mathrm{PMN} \%$ of $70 \%$, although a cut-off of $80 \%$ was validated in a cohort of 684 infected cases and 820 aseptic revisions for the latter $[82,83]$. The discrepancies in proposed cut-offs between studies may be due to differences in the type of infections included (acute / chronic) and/or how the control groups without an infection are defined (e.g., exclusion or inclusion of coinflammatory conditions, metallosis).

\section{Conclusion}

The synovial WBC count result is only one of several diagnostic results that may lead to a definite diagnosis of PJI. A threshold of $>3,000$ cells $/ \mu \mathrm{L}$ and a PMN\% of $>70$ has been recently suggested [84]. Most studies, however, have suggested WBC count cut-off values ranging from 1,100 to 4,200 cells $/ \mu \mathrm{L}$ and $60 \%$ to $89 \%$ for PMN\% for the diagnosis of PJI which may be due to differences in included infections (acute and chronic) and the defined control group.

In order to improve the diagnostic accuracy providers should pay attention to pre-analytic steps, the joint site, comorbidities and conditions that can mimic PJI.

The vast majority of studies on the subject of this review stem from chronic hip and knee PJI. A lower WBC count cut-off than 3000 cells $/ \mu \mathrm{L}$ may be applied for knees to diagnose a PJI. The synovial WBC count in inflammatory conditions such crystal-induced arthritis and metallosis may return false-positive results, but its diagnostic accuracy does not increase 
when providing a higher cut-off value.

For a single patient, it is important to take these variables into account, and, from a clinical point of view, PJI should be suspected when the cell count result falls above a "range" of cell count values instead of a precise cut-off value.

\section{Abbreviations}

CI: confidence interval; EDTA: ethylenediaminetetraacetate; ICM: International Consensus Meeting; MoM: metal-on-metal; MSIS: Musculoskeletal Infection Society; PJI: periprosthetic joint infection; $\mathrm{PMN} \%$ : percentage of polymorphonuclear leucocytes; RBC: red blood cell; THA: total hip arthroplasty; TKA: total knee arthroplasty; WBC: white blood cell.

\section{Acknowledgment}

Barbara Every, ELS, of BioMedical Editor, St. Albert, Alberta, Canada, provided English language editing.

\section{Author's contributions}

$\mathrm{KO}$ reviewed the literature and wrote the manuscript.

CS critically reviewed the manuscript.

MWB and PS supervised $\mathrm{KO}$ and revised the manuscript.

AMK reviewed the manuscript particularly concerning the pre-analytical steps.

\section{Competing interests}

The authors have declared that no competing interest exists.

\section{References}

1. Anguita-Alonso P, Hanssen AD, Patel R. Prosthetic joint infection. Expert Rev. Anti-Inf. 2005; 3: 797-804.

2. Gundtoft PH, Overgaard S, Schønheyder HC, et al. The "true" incidence of surgically treated deep prosthetic joint infection after 32,896 primary total hip arthroplasties: a prospective cohort study. Acta Orthop 2015; 86: 326-34.

3. Parvizi J, Fassihi SC, Enayatollahi MA. Diagnosis of Periprosthetic Joint Infection Following Hip and Knee Arthroplasty. Orthop. Clin. North Am. 2016; 47: 505-15.

4. Ahmad SS, Becker R, Chen AF, et al. EKA survey: diagnosis of prosthetic knee joint infection. Knee Surg. Sports Traumatol. Arthrosc. 2016; 24: 3050-5.

5. Huerfano E, Bautista M, Huerfano $M$, et al. Screening for Infection Before Revision Hip Arthroplasty: A Meta-analysis of Likelihood Ratios of Erythrocyte Sedimentation Rate and Serum C-reactive Protein Levels. J Am Acad Orthop Surg 2017; 25: 809-17.

6. Corvec S, Portillo ME, Pasticci BM, et al. Epidemiology and new developments in the diagnosis of prosthetic joint infection. Int. J. Artif. Organs 2012; 35: 923-34.

7. Pérez-Prieto D, Portillo ME, Puig-Verdié L, et al. C-reactive protein may misdiagnose prosthetic joint infections, particularly chronic and low-grade infections. Int Orthop 2017; 41: 1315-9.

8. Gundtoft $\mathrm{PH}$, Pedersen $\mathrm{AB}$, Varnum $\mathrm{C}$, et al. Increased Mortality After Prosthetic Joint Infection in Primary THA. Clin. Orthop. Relat. Res. 2017; 475: 2623-31.

9. Jacobs AME, Bénard M, Meis JF, et al. The unsuspected prosthetic joint infection : incidence and consequences of positive intra-operative cultures in presumed aseptic knee and hip revisions. Bone Joint J 2017; 99-B: 1482-9.

10. Parvizi J, Tan TL, Goswami K, et al. The 2018 Definition of Periprosthetic Hip and Knee Infection: An Evidence-Based and Validated Criteria. J. Arthroplasty 2018; 33: 1314.e2.
11. Ting NT, Della Valle CJ. Diagnosis of Periprosthetic Joint Infection-An Algorithm-Based Approach. J Arthroplasty 2017; 32: 2047-50.

12. Parvizi J, Gehrke T, Chen AF. Proceedings of the International Consensus on Periprosthetic Joint Infection. Bone Joint J. 2013; 95-B: 1450-2.

13. Tande AJ, Patel R. Prosthetic joint infection. Clin. Microbiol. Rev. 2014; 27: 302-45.

14. AUSTEN FK, CALKINS E. Serial Studies of Synovial Fluid in evaluating Intra-Articular Agents. Annals of the rheumatic diseases 1955; 14: 283-7.

15. Shmerling RH, Delbanco TL, Tosteson AN, et al. Synovial fluid tests. What should be ordered? JAMA 1990; 264: 1009-14.

16. Margaretten ME, Kohlwes J, Moore D, et al. Does this adult patient have septic arthritis? JAMA 2007; 297: 1478-88.

17. Duff GP, Lachiewicz PF, Kelley SS. Aspiration of the knee joint before revision arthroplasty. Clin. Orthop. Relat. Res. 1996: 132-9.

18. Spangehl MJ, Masri BA, O'Connell JX, et al. Prospective analysis of preoperative and intraoperative investigations for the diagnosis of infection at the sites of two hundred and two revision total hip arthroplasties. J Bone Joint Surg Am 1999; 81: 672-83.

19. Kersey R, Benjamin J, Marson B. White blood cell counts and differential in synovial fluid of aseptically failed total knee arthroplasty. J Arthroplasty 2000; 15: 301-4.

20. Mason JB, Fehring TK, Odum SM, et al. The value of white blood cell counts before revision total knee arthroplasty. J Arthroplasty 2003; 18: 1038-43.

21. Trampuz A, Hanssen AD, Osmon DR, et al. Synovial fluid leukocyte count and differential for the diagnosis of prosthetic knee infection. Am. J. Med. 2004; 117: 556-62.

22. Schinsky MF, Della Valle CJ, Sporer SM, et al. Perioperative testing for joint infection in patients undergoing revision total hip arthroplasty. J Bone Joint Surg Am 2008; 90: 1869-75.

23. Margaretten ME, Kohlwes J, Moore D, et al. Does this adult patient have septic arthritis? JAMA 2007; 297: 1478-88

24. Elsissy PG, Stevens WT, Ellsworth B, et al. Cell count and differential of aspirated fluid in the diagnosis of infection at the site of total knee arthroplasty. J Bone Joint Surg Am 2010; 92: 1314

25. Johnson SM, Saint John BE, Dine AP. Local anesthetics as antimicrobial agents: a review. Surg Infect (Larchmt) 2008; 9: 205-13.

26. Shahi A, Deirmengian C, Higuera C, et al. Premature Therapeutic Antimicrobial Treatments Can Compromise the Diagnosis of Late Periprosthetic Joint Infection. Clin. Orthop. Relat. Res. 2015; 473: 2244-9.

27. Zahar A, Lausmann C, Cavalheiro C, et al. How Reliable Is the Cell Count Analysis in the Diagnosis of Prosthetic Joint Infection? J Arthroplasty 2018.

28. Salinas M, Rosas J, Iborra J, et al. Comparison of manual and automated cell counts in EDTA preserved synovial fluids. Storage has little influence on the results. Ann. Rheum. Dis. 1997; 56: 622-6.

29. Roquet I, Hendrick S, Carmalt JL. The effect of blood contamination on equine synovial fluid analysis. Vet Comp Orthop Traumatol 2012; 25: 460-5

30. Ghanem E, Houssock C, Pulido L, et al. Determining "true" leukocytosis in bloody joint aspiration. J. Arthroplasty 2008; 23: 182-7.

31. de Jonge R, Brouwer R, Smit M, et al. Automated counting of white blood cells in synovial fluid. Rheumatology (Oxford) 2004; 43: 170-3.

32. Sugiuchi $\mathrm{H}$, Ando $\mathrm{Y}$, Manabe M, et al. Measurement of total and differential white blood cell counts in synovial fluid by means of an automated hematology analyzer. J. Lab. Clin. Med. 2005; 146: 36-42.

33. Sandhaus LM. Body fluid cell counts by automated methods. Clin. Lab. Med. 2015; 35: 93-103.

34. Schumacher HR, Sieck MS, Rothfuss S, et al. Reproducibility of synovial fluid analyses. A study among four laboratories. Arthritis Rheum. 1986; 29: 770-4.

35. Bingham J, Clarke $\mathrm{H}$, Spangehl $\mathrm{M}$, et al. The alpha defensin-1 biomarker assay can be used to evaluate the potentially infected total joint arthroplasty. Clin. Orthop. Relat. Res. 2014; 472: 4006-9.

36. Choi H-, Agrawal K, Bedair H. The diagnostic thresholds for synovial fluid analysis in late periprosthetic infection of the hip depend on the duration of symptoms. Bone Jt. J. 2016; 98-B: 1355-9.

37. Cipriano CA, Brown NM, Michael AM, et al. Serum and synovial fluid analysis for diagnosing chronic periprosthetic infection in patients with inflammatory arthritis. J Bone Joint Surg Am 2012; 94: 594-600.

38. Dinneen A, Guyot A, Clements J, et al. Synovial fluid white cell and differential count in the diagnosis or exclusion of prosthetic joint infection. Bone Joint J. 2013; 95-B: 554-7.

39. Higuera CA, Zmistowski B, Malcom T, et al. Synovial fluid cell count for diagnosis of chronic periprosthetic hip infection. J. Bone Jt. Surg. Am. Vol. 2017; 99: 753-9.

40. Kwon Y, Antoci V, Leone WA, et al. Utility of Serum Inflammatory and Synovial Fluid Counts in the Diagnosis of Infection in Taper Corrosion of Dual Taper Modular Stems. J Arthroplasty 2016; 31: 1997-2003.

41. Omar M, Reichling M, Ettinger M, et al. Synovial C-reactive protein as marker for chronic periprosthetic infection in total hip arthroplasty. Langenbeck's Arch. Surg. 2014; 399: 917.

42. Parvizi J, Ghanem E, Menashe $\mathrm{S}$, et al. Periprosthetic infection: What are the diagnostic challenges? J. Bone Jt. Surg. Ser. A 2006; 88: 138-47.

43. Schinsky MF, Della Valle CJ, Sporer SM, et al. Perioperative testing for joint infection in patients undergoing revision total hip arthroplasty. J. Bone Jt. Surg. Ser. A 2008; 90: 1869-75.

44. Wyles CC, Larson DR, Houdek MT, et al. Utility of synovial fluid aspirations in failed metal-on-metal total hip arthroplasty. J. Arthroplasty 2013; 28: 818-23. 
45. Yi PH, Cross MB, Moric M, et al. The 2013 frank stinchfield award: Diagnosis of infection in the early postoperative period after total hip arthroplasty. Clin. Orthop. Relat. Res. 2014; 472: 424-9.

46. Balato G, Franceschini V, Ascione T, et al. Diagnostic accuracy of synovial fluid, blood markers, and microbiological testing in chronic knee prosthetic infections. Arch. Orthop. Trauma. Surg. 2018; 138: 165-71.

47. Bedair H, Ting N, Jacovides C, et al. The Mark Coventry Award: diagnosis of early postoperative TKA infection using synovial fluid analysis. Clin. Orthop. Relat. Res. 2011; 469: 34-40.

48. Chalmers PN, Walton D, Sporer SM, et al. Evaluation of the role for synovial aspiration in the diagnosis of aseptic loosening after total knee arthroplasty. J. Bone Jt. Surg. Am. Vol. 2015; 97: 1597-603.

49. Ghanem E, Parvizi J, Burnett RSJ, et al. Cell count and differential of aspirated fluid in the diagnosis of infection at the site of total knee arthroplasty. J Bone Joint Surg Am 2008; 90: 1637-43.

50. Mason JB, Fehring TK, Odum SM, et al. The value of white blood cell counts before revision total knee arthroplasty. J Arthroplasty 2003; 18: 1038-43.

51. Trampuz A, Hanssen AD, Osmon DR, et al. Synovial fluid leukocyte count and differential for the diagnosis of prosthetic knee infection. Am. J. Med. 2004; 117: 556-62.

52. Zmistowski B, Restrepo C, Huang R, et al. Periprosthetic joint infection diagnosis: a complete understanding of white blood cell count and differential. J Arthroplasty 2012; 27: 1589-93.

53. Qu X, Zhai Z, Liu X, et al. Evaluation of white cell count and differential in synovial fluid for diagnosing infections after total hip or knee arthroplasty. PLoS One 2014; 9: e84751.

54. Lee SC, Jung KA, Yoon JY, et al. Analysis of synovial fluid in culture-negative samples of suspicious periprosthetic infections. Orthopedics 2010; 33: 725.

55. De Fine M, Giavaresi G, Fini M, et al. The role of synovial fluid analysis in the detection of periprosthetic hip and knee infections: a systematic review and meta-analysis. Int. Orthop. 2018; 42: 983-94.

56. Jerosch J, Schneppenheim M. Management of infected shoulder replacement. Arch Orthop Trauma Surg 2003; 123: 209-14.

57. Piper KE, Jacobson MJ, Cofield RH, et al. Microbiologic diagnosis of prosthetic shoulder infection by use of implant sonication. J. Clin. Microbiol. 2009; 47: 1878-84

58. Strahm C, Zdravkovic V, Egidy C, et al. Accuracy of Synovial Leukocyte and Polymorphonuclear Cell Count in Patients with Shoulder Prosthetic Joint Infection. J Bone Jt Infect 2018; 3: 245-8.

59. Sendi P, Moor BK, Zumstein MA. Periprosthetic Joint Infection after Shoulder Arthroplasty. In: Zimmerli W, ed. Bone and Joint Infections. Chichester, UK: John Wiley \& Sons, Ltd, 2015:151-66.

60. Alrashidi Y, Galhoum AE, Wiewiorski M, et al. How To Diagnose and Treat Infection in Total Ankle Arthroplasty. Foot Ankle Clin 2017; 22: 405-23.

61. Somerson JS, Morrey ME, Sanchez-Sotelo J, et al. Diagnosis and Management of Periprosthetic Elbow Infection. J Bone Joint Surg Am 2015; 97: 1962-71.

62. Aletaha D, Neogi T, Silman AJ, et al. 2010 Rheumatoid arthritis classification criteria: an American College of Rheumatology/European League Against Rheumatism collaborative initiative. Arthritis Rheum. 2010; 62: 2569-81.

63. Qu X, Zhai Z, Liu X, et al. Evaluation of white cell count and differential in synovial fluid for diagnosing infections after total hip or knee arthroplasty. PLoS ONE 2014; 9: e84751.

64. Shohat N, Goswami K, Fillingham Y, et al. Diagnosing Periprosthetic Joint Infection in Inflammatory Arthritis: Assumption Is the Enemy of True Understanding. J Arthroplasty 2018.

65. Hexter AT, Hislop SM, Blunn GW, et al. The effect of bearing surface on risk of periprosthetic joint infection in total hip arthroplasty: a systematic review and meta-analysis. Bone Joint J 2018; 100-B: 134-42.

66. Yi PH, Cross MB, Moric M, et al. Do Serologic and Synovial Tests Help Diagnose Infection in Revision Hip Arthroplasty With Metal-on-metal Bearings or Corrosion? Clin. Orthop. Relat. Res. 2014; 473: 498-505.

67. Rompen JC, Kuiper-Geertsma DG, Verheyen CCPM. Gouty arthritis in a total knee prosthesis. Ned Tijdschr Geneeskd 2008; 152: 1117-9.

68. Soloway S, Tucker BS. Calcium Pyrophosphate Dihydrate Deposition Disease in a Knee With Total Joint Replacement. J Clin Rheumatol 2016; 22: 277.

69. Archibeck MJ, Rosenberg AG, Sheinkop MB, et al. Gout-induced arthropathy after total knee arthroplasty: a report of two cases. Clin. Orthop. Relat. Res. 2001: 377-82.

70. George MP, Ernste FC, Tande A, et al. Clinical Presentation, Management, and Prognosis of Pseudogout in Joint Arthroplasty: A Retrospective Cohort Study. J Bone Jt Infect 2019; 4: 20-6.

71. Zadaka A, Gioe T, Gertner E. Acute crystal-induced arthritis following arthroplasty. J Knee Surg 2010; 23: 17-20.

72. Partridge DG, Gordon A, Townsend R. False-positive synovial fluid alpha-defensin test in a patient with acute gout affecting a prosthetic knee. Eur J Orthop Surg Traumatol 2017; 27: 549-51.

73. Schulz BM, Watling JP, Vosseller JT, et al. Markedly elevated intra-articular white cell count caused by gout alone. Orthopedics 2014; 37: 739 .

74. Shah K, Spear J, Nathanson LA, et al. Does the presence of crystal arthritis rule out septic arthritis? J Emerg Med 2007; 32: 23-6.

75. Salt E, Wiggins AT, Rayens MK, et al. Moderating effects of immunosuppressive medications and risk factors for post-operative joint infection following total joint arthroplasty in patients with rheumatoid arthritis or osteoarthritis. Semin. Arthritis Rheum. 2017; 46: 423-9.
76. Rienmuller A, Borens O. Propionibacterium prosthetic joint infection: experience from a retrospective database analysis. Eur. J. Orthop. Surg. Traumatol. 2016; 26: 429-34.

77. Renz N, Mudrovcic S, Perka C, et al. Orthopedic implant-associated infections caused by Cutibacterium spp. - A remaining diagnostic challenge. PLoS ONE 2018; 13: e0202639.

78. Shields MV, Abdullah L, Namdari S. The challenge of Propionibacterium acnes and revision shoulder arthroplasty: A review of current diagnostic options. J. Shoulder Elbow Surg. 2016; 25: 1034-40.

79. Kheir MM, Tan TL, Shohat N, et al. Routine Diagnostic Tests for Periprosthetic Joint Infection Demonstrate a High False-Negative Rate and Are Influenced by the Infecting Organism. J Bone Joint Surg Am 2018; 100: 2057-65.

80. Renz N, Yermak K, Perka C, et al. Alpha Defensin Lateral Flow Test for Diagnosis of Periprosthetic Joint Infection: Not a Screening but a Confirmatory Test. J Bone Joint Surg Am 2018; 100: 742-50.

81. Lee YS, Koo K, Kim HJ, et al. Synovial Fluid Biomarkers for the Diagnosis of Periprosthetic Joint Infection: A Systematic Review and Meta-Analysis. J Bone Joint Surg Am 2017; 99: 2077-84.

82. Parvizi J, Tan TL, Goswami K, et al. The 2018 Definition of Periprosthetic Hip and Knee Infection: An Evidence-Based and Validated Criteria. J Arthroplasty 2018; 33: 1314.e2.

83. Shohat N, Bauer T, Buttaro M, et al. Hip and Knee Section, What is the Definition of a Periprosthetic Joint Infection (PJI) of the Knee and the Hip? Can the Same Criteria be Used for Both Joints?: Proceedings of International Consensus on Orthopedic Infections. J Arthroplasty 2019; 34: S327.

84. Shohat N, Bauer T, Buttaro $M$ et al. Hip and knee section, what is the definition of a Periprosthetic Joint Infection (PJI) of the knee and the hip? Can the same criteria be used for both joints?: proceedings of international consensus on orthopedic infections. J Arthroplasty 2019; 34(2S): S325-S327. 